\title{
EVALUACIÓN DE LA GESTIÓN DEL CONOCIMIENTO: UNA REVISIÓN SISTEMÁTICA DE LITERATURA
}

\author{
Por: Ernesto Galvis-Lista ${ }^{1}$ - Jenny Marcela Sánchez-Torres ${ }^{2}$
}

\section{RESUMEN}

La evaluación de la gestión del conocimiento (GC) es un tema importante para aquellas organizaciones que quieran saber "qué está pasando" con sus estrategias de GC. No obstante, no existe un consenso sobre qué evaluar y cómo evaluarlo. Por esta razón, el propósito del artículo es presentar una revisión sistemática de literatura de 43 artículos publicados en la última década. La revisión comprende un análisis cienciométrico básico y un análisis de contenido relacionado con varios aspectos de los modelos como su estructura, la función y objetivo de la evaluación, lo métodos de investigación utilizados, los sectores económicos de aplicación, y la ubicación de los aspectos evaluados respecto de una taxonomía de escuelas de pensamiento de la GC y una clasificación de las capacidades organizacionales de GC. Como principal hallazgo se muestra la predominancia del enfoque de GC como codificación de conocimiento. Además, se presentan varias brechas susceptibles de investigación futura.

Palabras clave: Gestión de Conocimiento, Modelos de Evaluación, Revisión Sistemática.

Clasificación JEL: M1 Business Administration - D8 Information, Knowledge, and Uncertainty.

1. Profesor Asociado - Universidad del Magdalena - Estudiante de Doctorado en Ingeniería - Sistemas y Computación - UNAL - egalvis@unimagdalena.edu.co

2. Profesora Asociada - Universidad Nacional de Colombia UNAL - jmsanchezt@unal.edu.co 


\title{
KNOWLEDGE MANAGEMENT EVALUATION: A SYSTEMATIC LITERATURE REVIEW
}

\author{
By: Ernesto Galvis-Lista - Jenny Marcela Sánchez-Torres
}

\begin{abstract}
The evaluation of knowledge management (KM) is an important concern for those organizations that want to know "what is happening" with their KM strategies. Nevertheless, there is no consensus on what to evaluate and how to evaluate it. For this reason, the purpose of this paper is to present a systematic literature review of 43 papers published within the last decade. The review is composed by a basic scientometric analysis and a content analysis. The content analysis is related to various aspects of the models of KM evaluation: their structure, function and purpose of the evaluation, the research methods used in the construction of the model, the industry of application, and the location of the evaluated aspects regarding to a taxonomy of KM schools of thought and a classification of the KM organizational capabilities. The main finding is the predominance of a knowledge codification approach. In addition, various gaps, susceptible for future research, are identified.
\end{abstract}

Keywords: Knowledge Management, Evaluation Models, Systematic Review.

JEL Classification: M1 Business Administration - D8 Information, Knowledge, and Uncertainty. 


\section{Introducción}

El campo de la Gestión del Conocimiento (GC) está en pleno desarrollo. En un análisis cienciométrico de la producción académica durante el período 1994 - 2008, elaborado por (Serenko, Bontis, Booker, Sadeddin, \& Hardie, 2010), se presentan conclusiones que demuestran el avance del área. Allí, se destaca el hecho de que, a pesar de su corta historia, la GC está conformada por un amplio cuerpo de conocimiento en continuo crecimiento. Además, se evidencia que la producción se ha realizado por una amplia gama de personas e instituciones académicas y no académicas. También, (Serenko et al., 2010) identifican hechos que muestran la evolución de este campo de estudio, como el incremento del índice de coautoría, la rigurosidad de los métodos de investigación y el predominio en la producción hecha en entornos académicos.

En particular, la evaluación de la GC ha sido un tema de interés para investigadores y practicantes durante la última década. Hace parte de las temáticas identificadas por Dwivedi, Venkitachalam, Sharif, Al-Karaghouli, \& Weerakkody, (2011) como tendencias de investigación en los próximos años, específicamente sobre la efectividad en la GC y el soporte organizacional y tecnológico necesario para lograrla. Por tal razón, para construir una visión general sobre la evaluación de la GC se presenta esta revisión sistemática de literatura (RSL) que abarca aportes publicados en la literatura científica durante la última década. Así, el alcance de esta revisión se centró en buscar conclusiones para las siguientes preguntas de interés:

1. ¿Qué modelos existen para evaluar la GC en una organización?

2. ¿En qué escuelas de GC se ubica el contenido evaluado por los modelos?

3. ¿En qué sectores de aplicación fueron utilizados los modelos?

4. ¿Cuáles de los modelos se centran en la evaluación de procesos de GC?

5. ¿Cuáles modelos se aplicaron en la industria del software?

Para presentar la RSL desarrollada, este documento se estructuró de la siguiente forma. En la sección dos se presenta la fundamentación teórica necesaria para dar sentido al análisis de los modelos de evaluación revisados. El centro de esta fundamentación teórica es una propuesta de escuelas de pensamiento de la GC y una clasificación de las capacidades organizacionales de GC. En la sección tres se describe la metodología utilizada para realizar la RSL. En la sección cuatro se presenta el detalle de los resultados obtenidos con el análisis de los 43 artículos incluidos en la RSL. Al final, en la sección cinco se presentan las conclusiones del trabajo y por último, las referencias bibliográficas utilizadas.

\section{Fundamentación Teórica}

Al considerar el reciente surgimiento y la diversidad conceptual del campo de la GC, una forma de llegar a una primera noción sobre lo que significa GC es abordar el análisis a través de enfoques y escuelas de pensamiento. Por esto, se tomó la taxonomía de (Earl, 2001) como uno de los referentes base pues es un esquema detallado en donde el componente conceptual es sustentado con estu- 
dios empíricos como: seis casos de estudio en organizaciones, interacción con 20 gerentes de conocimiento sobre la implementación de iniciativas de GC, un taller sobre programas de GC con la red de gerentes de conocimiento del Reino Unido, y el análisis de programas de GC publicados en revistas académicas y profesionales. Además, es importante destacar que, aunque cada escuela representa un propósito o enfoque particular, no son competencia entre sí. Por el contrario, en la práctica se implementan estrategias y herramientas de varias escuelas (Earl, 2001).

Las escuelas Tecnocráticas se enfocan en herramientas de tecnologías de información (TI) que brindan soporte y, en cierto grado, condicionan a los miembros de la organización, en la realización de actividades de conocimiento. Las escuelas Económicas se orientan en aprovechar la relación entre los ingresos de la organización con la explotación de los activos de conocimiento y, en general, el capital intelectual que posee. Las escuelas Comportamentales se centran en la promoción y estímulo, para que las personas construyan, compartan y utilicen el conocimiento como un recurso. En la Tabla 1 se resume la taxonomía de Earl (2001).

\section{Tabla 1}

\section{Clasificación de las escuelas de GC.}

\begin{tabular}{|c|c|c|c|}
\hline Categoría & Escuela & Principio central & Ideas Fundamentales \\
\hline \multirow{3}{*}{ Tecnocráticas } & Sistemas & $\begin{array}{l}\text { Codificación de cono- } \\
\text { cimiento de dominio } \\
\text { específico. }\end{array}$ & $\begin{array}{l}\text { Codificación de conocimientos } \\
\text { especializados en bases de co- } \\
\text { nocimiento para ser utilizadas } \\
\text { por otros especialistas o perso- } \\
\text { nal calificado. }\end{array}$ \\
\hline & Cartográfica & $\begin{array}{l}\text { Conectividad de las } \\
\text { personas. }\end{array}$ & $\begin{array}{l}\text { Identificación y mapeo del } \\
\text { conocimiento organizacional } \\
\text { para su divulgación y utiliza- } \\
\text { ción, asegurando que las per- } \\
\text { sonas que poseen conocimien- } \\
\text { to en la organización están } \\
\text { accesibles por otros para ase- } \\
\text { sorías y consultas. }\end{array}$ \\
\hline & Ingeniería & $\begin{array}{l}\text { Flujos de conocimiento } \\
\text { para mejorar las capa- } \\
\text { cidades centrales de la } \\
\text { organización. }\end{array}$ & $\begin{array}{l}\text { Provisión de personal con sufi- } \\
\text { ciente conocimiento sobre su } \\
\text { trabajo. } \\
\text { Formalización de procesos de } \\
\text { provisión de conocimiento } \\
\text { contextual y de mejores prácti- } \\
\text { cas al personal administrativo y } \\
\text { gerencial. }\end{array}$ \\
\hline
\end{tabular}




\begin{tabular}{|c|c|c|c|}
\hline Categoría & Escuela & Principio central & Ideas Fundamentales \\
\hline Económicas & Comercial & $\begin{array}{l}\text { Comercialización de la } \\
\text { propiedad intelectual o } \\
\text { de conocimiento. }\end{array}$ & $\begin{array}{l}\text { La protección y explotación de } \\
\text { los activos intelectuales o de } \\
\text { conocimiento de la organiza- } \\
\text { ción para producir ingresos y } \\
\text { rentas. }\end{array}$ \\
\hline \multirow{3}{*}{$\begin{array}{c}\text { Comporta- } \\
\text { mentales }\end{array}$} & $\begin{array}{l}\text { Organiza- } \\
\text { cional }\end{array}$ & $\begin{array}{l}\text { Incremento de la co- } \\
\text { nectividad entre los } \\
\text { trabajadores de cono- } \\
\text { cimiento. }\end{array}$ & $\begin{array}{l}\text { Uso de estructuras o redes or- } \\
\text { ganizacionales para compartir, } \\
\text { o poner en común el conoci- } \\
\text { miento. } \\
\text { Comunidades en donde se } \\
\text { intercambia y comparte el co- } \\
\text { nocimiento, en forma no ru- } \\
\text { tinaria o poco convencional, } \\
\text { personal, y poco estructurada. }\end{array}$ \\
\hline & Espacial & $\begin{array}{l}\text { Diseño de espacios fí- } \\
\text { sicos para fomentar el } \\
\text { contacto y la actividad } \\
\text { de conocimiento. }\end{array}$ & $\begin{array}{l}\text { Diseño y uso de los espacios } \\
\text { para facilitar el intercambio de } \\
\text { conocimiento. } \\
\text { Fomentar la socialización como } \\
\text { medio de intercambio de co- } \\
\text { nocimiento. }\end{array}$ \\
\hline & Estratégica & $\begin{array}{l}\text { Toma de conciencia so- } \\
\text { bre las posibilidades de } \\
\text { creación de valor al re- } \\
\text { conocer al conocimien- } \\
\text { to como un recurso. }\end{array}$ & $\begin{array}{l}\text { El conocimiento como una di- } \\
\text { mensión esencial de la estrate- } \\
\text { gia competitiva. } \\
\text { La firma es conceptualizada } \\
\text { como un negocio de conoci- } \\
\text { miento. } \\
\text { Las acciones de gestión del co- } \\
\text { nocimiento son variadas y pue- } \\
\text { den enmarcarse en las demás } \\
\text { escuelas. }\end{array}$ \\
\hline
\end{tabular}

Fuente: Taxonomía de Earl, 2001.

El segundo referente es el trabajo de Gold, Malhotra, \& Segars (2001), quienes argumentan que las organizaciones deben aprovechar el conocimiento existente y crear conocimiento para competir en el mercado. Para lograr esto, las organizaciones deben desarrollar dos tipos de capacidades: capacidades de infraestructura de conocimiento y capacidades de procesos de conocimiento. Las capacidades de infraestructura permiten la maximización del capital social, entendido como "la suma de los recursos actuales y potenciales que están embebidos dentro de, disponibles a través de, y derivados de la red de relaciones que posee una unidad social" (Gold et al., 2001). De forma complementaria, las capacidades de procesos son los elementos dinámicos que aprovechan las capacidades de infraestructura para hacer del conocimiento un recurso organizacional activo. En la Tabla 2 se sintetizan los planteamientos de esta propuesta. 
Tabla 2

Capacidades de GC: Infraestructura y Procesos

\begin{tabular}{|c|c|c|}
\hline Categorías & Capacidades & Principio central \\
\hline \multirow{3}{*}{ Infraestructura } & Tecnología & $\begin{array}{l}\text { Los sistemas de } \mathrm{TI} \text { determinan la forma en que el cono- } \\
\text { cimiento es transferido y accedido. }\end{array}$ \\
\hline & Estructura & $\begin{array}{l}\text { Las estructuras organizacionales, formales e informales, } \\
\text { pueden inhibir o facilitar la interacción entre las perso- } \\
\text { nas, aspecto esencial en la GC. }\end{array}$ \\
\hline & Cultura & $\begin{array}{l}\text { La cultura organizacional debe soportar y potenciar las } \\
\text { actividades de conocimiento. }\end{array}$ \\
\hline \multirow{4}{*}{ Procesos } & Adquisición & $\begin{array}{l}\text { Ubicación y adquisición de conocimiento o creación de } \\
\text { conocimiento a través de la colaboración entre indivi- } \\
\text { duos y socios de negocios. }\end{array}$ \\
\hline & Conversión & $\begin{array}{l}\text { El conocimiento debe ser organizado y estructurado de } \\
\text { forma tal que se facilite su distribución y uso dentro de } \\
\text { la organización. }\end{array}$ \\
\hline & Aplicación & $\begin{array}{l}\text { El conocimiento debe utilizarse para ajustar la dirección } \\
\text { estrategia, resolver nuevos problemas y mejorar la efi- } \\
\text { ciencia. }\end{array}$ \\
\hline & Protección & $\begin{array}{l}\text { El conocimiento debe ser protegido de usos inapropia- } \\
\text { dos, o de explotación no autorizada. }\end{array}$ \\
\hline
\end{tabular}

Fuente: Gold, Malhotra, \& Segars, 2001.

\section{Metodología}

En esta revisión se utilizó el método propuesto por B. A. Kitchenham, Dybå, \& Jørgensen (2004); B. Kitchenham \& Charters (2007); B. Kitchenham et al (2009, 2010). Este método inicia con la elaboración de un protocolo para la revisión cuyo propósito es la especificación de preguntas de interés, estrategias de búsqueda, criterios de inclusión, criterios para evaluar la calidad de los estudios, y el procedimiento de extracción y síntesis de datos. Por tanto, para cumplir con el propósito de esta RSL se propusieron las preguntas de interés enumeradas en la introducción de este artículo.

La definición de estrategias de búsquedas comprende dos elementos: la determinación de las fuentes a consultar y de las expresiones de búsqueda que se utilizan en los motores de búsqueda de las fuentes seleccionadas. Las fuentes utilizadas en la revisión se seleccionaron por ser bases de datos que recogen la mayoría de la producción científica en el tema: SCOPUS, ISI Web of Science, Sciencedirect, IEEE Explorer, ACM DL, Emerald, Springerlink, y EI Compendex.

Por otra parte, la construcción de las ecuaciones de búsqueda se desarrolló realizando prototipos de términos y expresiones. El resultado de este proceso iterativo de construcción dio como resultado una expresión de búsqueda con palabras clave, sintagmas y conectores booleanos. Es importante destacar que las expresiones de 
búsqueda se construyeron partiendo de cada una de las preguntas de interés para esta revisión. Para esto, se tomaron palabras clave y sintagmas a partir de las preguntas. Luego, se fueron refinando, a partir de la evaluación de los resultados de cada expresión, con la incorporación de sinónimos y alternativas para la escritura de los términos, palabras clave obtenidas de artículos relevantes revisados previamente, y la incorporación de filtros proporcionados por las diferentes fuentes, como los relativos al tipo de artículo, a las fechas de publicación, y referencias. La ecuación de búsqueda definitiva fue la siguiente:

("knowledge management capability" OR "knowledge management capabilities" OR "capability of knowledge management” OR "capabilities of knowledge management" OR "capability for knowledge management" OR "knowledge management capacity” OR "capacity of knowledge management” OR "knowledge management capacities" OR "capacities of knowledge management" OR "Knowledge management maturity" OR "maturity of knowledge management" OR "Knowledge management evaluation" OR "evaluation of knowledge management" OR "evaluating knowledge management" OR "knowledge management assessment” OR "assessment of knowledge management” OR "assessing knowledge management" OR "knowledge management measurement" OR "measurement of knowledge management" OR "measuring knowledge management" OR "knowledge management metrics" OR "metrics of knowledge management" OR "metrics for knowledge management" OR "knowledge management performance" OR "performance of knowledge management" OR "KM capability" OR "KM capabilities" OR "capability of KM" OR "capability for KM" OR "capabilities of KM" OR "KM capacity” OR "capacity of KM" OR "KM capacities" OR "capacities of KM" OR "KM maturity" OR "maturity of KM" OR "KM evaluation" OR "evaluation of KM" OR "evaluating KM" OR "KM assessment" OR "assessment of KM" OR "assessing KM" OR "KM measurement" OR "measurement of KM" OR "measuring KM" OR "KM metrics" OR "metrics of KM" OR "metrics for KM” OR "km performance” OR "performance of km”) OR (("knowledge management” OR "KM”) AND ("organizational capabilities” OR "organizational capability" OR "enterprise capabilities" OR "enterprise capability”)) OR ("knowledge management systems" AND ("evaluation" OR assessment OR measurement)).

El resultado del uso de la ecuación de búsqueda en las ocho fuentes fueron 2503 registros bibliográficos potencialmente relevantes. Luego, los registros recopilados fueron sometidos a un proceso de depuración para eliminar duplicados, obteniéndose 754. Posteriormente, se aplicaron como criterios de inclusión en el análisis dos aspectos: que el artículo presente un modelo en el cual se describa el contenido a evaluar, y que su construcción sea resultado de investigación y no de una opinión de un experto o del reporte de un caso particular, obteniendo como resultado 177 registros. Seguidamente se realizó una evaluación de calidad de cada documento, que consistió en la revisión del texto completo para identificar si el artículo trataba aspectos como el contenido del modelo, la metodología utilizada para construirlo, la aplicación y los resultados de la misma, y el contexto de aplicación en el que se llevó a cabo el trabajo. El resultado de esta evaluación de calidad fue la selección de 43 artículos que finalmente fueron los estudiados en esta RSL. En la Tabla 3 se 
presentan las referencias bibliográficas de los artículos seleccionados ordenados por el año de publicación.

Tabla 3

Artículos seleccionados para la revisión

\begin{tabular}{|c|c|c|}
\hline Año & $\mathbf{N}$ & Referencias \\
\hline 2001 & 1 & (J.-H. Lee \& Kim, 2001) \\
\hline 2004 & 1 & (Smits \& De Moor, 2004) \\
\hline 2005 & 3 & (Freeze \& Kulkarni, 2005; Hung \& Shou, 2005; K. C. Lee, Lee, \& Kang, 2005) \\
\hline 2006 & 2 & (Anantatmula \& Shivraj, 2006; Pee, Kankanhalli, \& Teah, 2006) \\
\hline 2007 & 6 & $\begin{array}{l}\text { (Chua \& Goh, 2007; Fang, Peide, \& Xin, 2007; Holt, Bartczak, Clark, \& Trent, } \\
\text { 2007; Huang, Chen, \& Yieh, 2007; Zaim, Tatoglu, \& Zaim, 2007; Q.-P. Zhang \& } \\
\text { Shan, 2007) }\end{array}$ \\
\hline 2008 & 6 & $\begin{array}{l}\text { (Chua \& Goh, 2008; Lan, Zhong, \& Dai, 2008; Li \& Li, 2008; Mehta, 2008; Suo, } \\
\text { Wang, Dong, \& Zhao, 2008; Wei \& Bi, 2008) }\end{array}$ \\
\hline 2009 & 8 & $\begin{array}{l}\text { (Chen, Huang, \& Cheng, 2009; Fan, Feng, Sun, \& Ou, 2009; Gourova, Anton- } \\
\text { ova, \& Todorova, 2009; Hsieh, Lin, \& Lin, 2009; J. Wang, Fang, \& Wang, 2009; } \\
\text { Wen, 2009; Zheng \& Hu, 2009; Z.-H. Zhu \& Xue, 2009) }\end{array}$ \\
\hline 2010 & 13 & $\begin{array}{l}\text { (Chin, Lo, \& Leung, 2010; Goldoni \& Oliveira, 2010; Jafari, Rezaeenour, Akha- } \\
\text { van, \& Fesharaki, 2010; Khatibian, Gholoi Pour, \& Abedi Jafari, 2010; Kur- } \\
\text { niawati \& Anthony, 2010; Qi \& Liu, 2010; Razi \& Karim, 2010; Tseng, 2010; Y. } \\
\text { Wang \& Zheng, 2010; Xia, Pan, Chen, \& Zhao, 2010; Yang, 2010; H. Zhang \& } \\
\text { He, 2010; X.-Y. Zhu \&Wu, 2010) }\end{array}$ \\
\hline 2011 & 3 & (Goonesekera \& Zyngier, 2011; Hung, Chou, \& Tzeng, 2011; Tseng, 2011) \\
\hline
\end{tabular}

Fuente: Elaboración propia.

El origen de los artículos seleccionados, determinado a partir de la ubicación geográfica de las instituciones a las que están afiliados los autores mostró una alta diversidad. Como se ve en la Figura 1, se encontró que la mayoría de los documentos revisados provenían de China, con 17 artículos, seguida por Taiwán, Estados Unidos, Singapur y Corea del Sur. Solamente se identificaron tres documentos de Europa y uno de América Latina, específicamente de Brasil.

Por último, para la extracción y síntesis de datos se construyó una hoja de cálculo en Excel para registrar, el objeto y la función de la evaluación, el método usado para la construcción del modelo, si se presenta un modelo de referencia para la evaluación, el método de evaluación, si se proponen niveles de madurez, el sector de la industria en que se aplicó, si se tuvieron en cuenta características propias de la organización en que se aplicó, la ubicación del contenido evaluado en las escuelas de GC y en las capacidades de GC descritas en la fundamentación teórica, y la identificación de fortalezas y limitaciones de los modelos. 
Figura 1

Países de origen de los artículos incluidos en la revisión

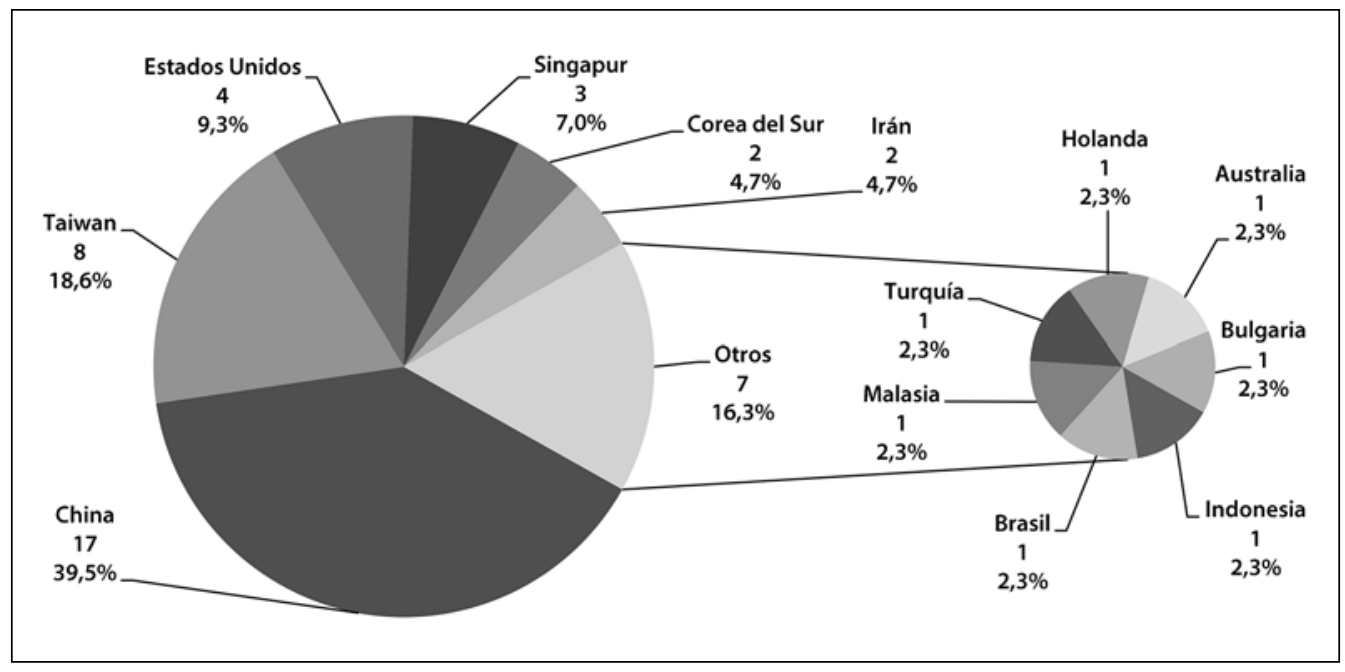

Fuente: Elaboración propia.

\section{Resultados}

La descripción de los resultados obtenidos para dar respuesta a las preguntas de interés inicia con algunos aspectos generales que permiten caracterizar los modelos de evaluación de GC. Esto apunta a responder la primera pregunta de interés: ¿Qué modelos existen para evaluar la GC en una organización? En primer lugar, se presenta el análisis sobre el objeto a evaluar, pues los modelos podrían orientarse a evaluar aspectos como: (1) la disposición de una organización para implementar la GC, (2) los resultados obtenidos con la implementación de la GC, (3) la forma en que están implementados los procesos de GC, y (4) una mezcla que incluye procesos, resultados e impacto de la GC sobre el desempeño organizacional. Aquí se encontró que el $69.8 \%$ de los modelos se enfocaban en evaluar los resultados, el $18.6 \%$ a los procesos, y el 4,7\% a la disposición para implementar la GC.

Luego, se analizaron los modelos de acuerdo a la función de la evaluación. Para esto se definieron dos tipos de evaluación, la sumativa que se centra en obtener valoraciones sobre el desempeño, y la formativa, que se usa para identificar aspectos a mejorar. La diferencia en la función de la evaluación no está determinada por el contenido a evaluar, sino en el uso que se le da al resultado de la misma. Aquí, se encontró que el $58.1 \%$ de los modelos se orientaban a una función sumativa para la evaluación, y el restante $41.9 \%$ una función formativa. Además, incluyendo el objeto de la evaluación dentro del análisis, se encontró que el $80 \%$ de los modelos enfocados a evaluar resultados asumían la función sumativa de la evaluación y el 20\% una función formativa. Para los otros objetos de evaluación, la mayoría de modelos asumían la función formativa de la evaluación. 
Luego, se construyó el análisis sobre la estructura de los modelos, en donde se encontró que el 25,6\% tienen una estructura con múltiples criterios organizados jerárquicamente o en red, aplicando el enfoque de análisis de decisiones con múltiples criterios de la investigación de operaciones. El 23,3\% utiliza técnicas de valoraciones con base en conjuntos difusos y el 23,3\% proponen conjuntos de indicadores cuantitativos con estructura simple y pesos equivalentes para los criterios de evaluación. Además, el 14\% tiene una estructura de niveles de madurez. En la Tabla 4, se presenta la distribución de acuerdo a la estructura.

Tabla 4

Estructura de los modelos de evaluación

\begin{tabular}{|l|c|}
\hline \multicolumn{1}{|c|}{ Estructura de la Evaluación } & Frecuencia \\
\hline Modelos cuantitativos basados en análisis de decisiones con múltiples criterios & 11 \\
\hline Modelos cuantitativos de evaluación por gradación difusa de criterios & 10 \\
\hline Indicadores cuantitativos simples & 10 \\
\hline Niveles de Madurez & 6 \\
\hline Otros métodos estadísticos de análisis de datos & 3 \\
\hline Cuadro de mando & 2 \\
\hline Indicadores cualitativos & 1 \\
\hline
\end{tabular}

Fuente: Elaboración propia.

Sumado a esto, como se ve en la Tabla 5, la mayoría de los modelos fueron construidos a partir de revisión de literatura y luego validados en contextos de práctica. Sin embargo, también se ve el uso de métodos empíricos, como los estudios de caso y grupos focales.

Tabla 5

Métodos de investigación empleados en la construcción de los modelos

\begin{tabular}{|l|c|}
\hline \multicolumn{1}{|c|}{ Principal Método de investigación } & Cantidad \\
\hline Revisión de Literatura & 31 \\
\hline Estudio de Caso & 6 \\
\hline Grupos Focales & 5 \\
\hline Modelado Matemático & 1 \\
\hline
\end{tabular}

Fuente: Elaboración propia.

El segundo elemento de indagación fue la ubicación del contenido de la evaluación dentro de las escuelas de GC (Earl, 2001). En este sentido, se identificó que en los 43 modelos estudiados, el contenido de la evaluación está organizado en categorías, y en algunos casos, esta organización tiene varios niveles de desagregación. Esas categorías y subcategorías fueron revisadas para determinar si el 
modelo incluía o no elementos de cada escuela de GC. En este sentido, como se observa en la Tabla 6, 33 de los 43 modelos (76.7\%) incluyen aspectos de la escuela organizacional. Luego se encuentran las escuelas de Sistemas y de Ingeniería, que están incluidas en $31(72.1 \%)$ y 30 (69.8\%) modelos respectivamente. Luego está la escuela Comercial con 19 modelos (44.2\%) y la escuela Estratégica con 12 (27.9\%). Por último, se encuentra la escuela Cartográfica, que está incluida únicamente en un modelo. Es de notar que los planteamientos de la escuela Espacial no hacen parte del contenido evaluado.

Tabla 6

Clasificación de los modelos respecto de las escuelas de GC

\begin{tabular}{|l|c|}
\hline \multicolumn{1}{|c|}{ Escuela } & Frecuencia \\
\hline Organizacional & 33 \\
\hline Sistemas & 31 \\
\hline Ingeniería & 30 \\
\hline Comercial & 19 \\
\hline Estratégica & 12 \\
\hline Cartográfica & 1 \\
\hline
\end{tabular}

Fuente: Elaboración propia.

Por otra parte, tomando como base el trabajo de Gold et al (2001), sobre capacidades organizacionales de GC, se realizó la clasificación del contenido evaluado mapeando las categorías propuestas en cada modelo con las tres capacidades de infraestructura de conocimiento (tecnología, estructura y cultura organizacional), y las cuatro capacidades de procesos de conocimiento (adquisición, conversión, aplicación, y protección del conocimiento). En la Tabla 7 se presenta el resultado de este análisis.

Tabla 7

Clasificación de los modelos respecto de las capacidades de GC

\begin{tabular}{|l|l|c|}
\hline \multicolumn{1}{|c|}{ Tipo } & \multicolumn{1}{|c|}{ Capacidades de GC } & Frecuencia \\
\hline \multirow{4}{*}{ Infraestructura } & Estructura & 27 \\
\cline { 2 - 3 } & Tecnología & 21 \\
\cline { 2 - 3 } & Cultura & 16 \\
\hline \multirow{4}{*}{ Procesos } & Adquisición & 34 \\
\cline { 2 - 3 } & Aplicación & 29 \\
\cline { 2 - 3 } & Conversión & 16 \\
\cline { 2 - 3 } & Evaluación & 16 \\
\hline
\end{tabular}

Fuente: Elaboración propia. 
El tercer elemento de indagación fue la identificación de los sectores de la industria en donde fueron construidos o aplicados los modelos. Aquí se encontró gran diversidad de sectores que incluyen la industria manufacturera de diferentes tipos, el sector de servicios, la educación e investigación, y el sector gubernamental. Como se ve en la Figura 2, en el 25,6 \% no se hace explícito el sector de aplicación. Además, se evidencia que dentro de los modelos en los cuales se enuncia el sector de aplicación, el mayor porcentaje está en la industria del software, con un 14\%. Los siguientes cinco sectores con mayor aplicación de los modelos son los de manufactura, gobierno, educación superior, servicios de TI y, servicios financieros, con $6.98 \%$ cada uno. Sin embargo, si se suman los porcentajes del sector de software y los servicios de TI, dada su afinidad, se evidencia que hay un gran interés en la evaluación de GC en comparación con otras industrias. Además, de los seis sectores con mayor porcentaje, solamente uno, el de manufactura, se ubica en la franja de industrias tradicionales de transformación de materias primas en productos, y los demás, son organizaciones que ofrecen servicios basados en el conocimiento.

Figura 2

Sectores de aplicación de los modelos de evaluación de GC estudiados.

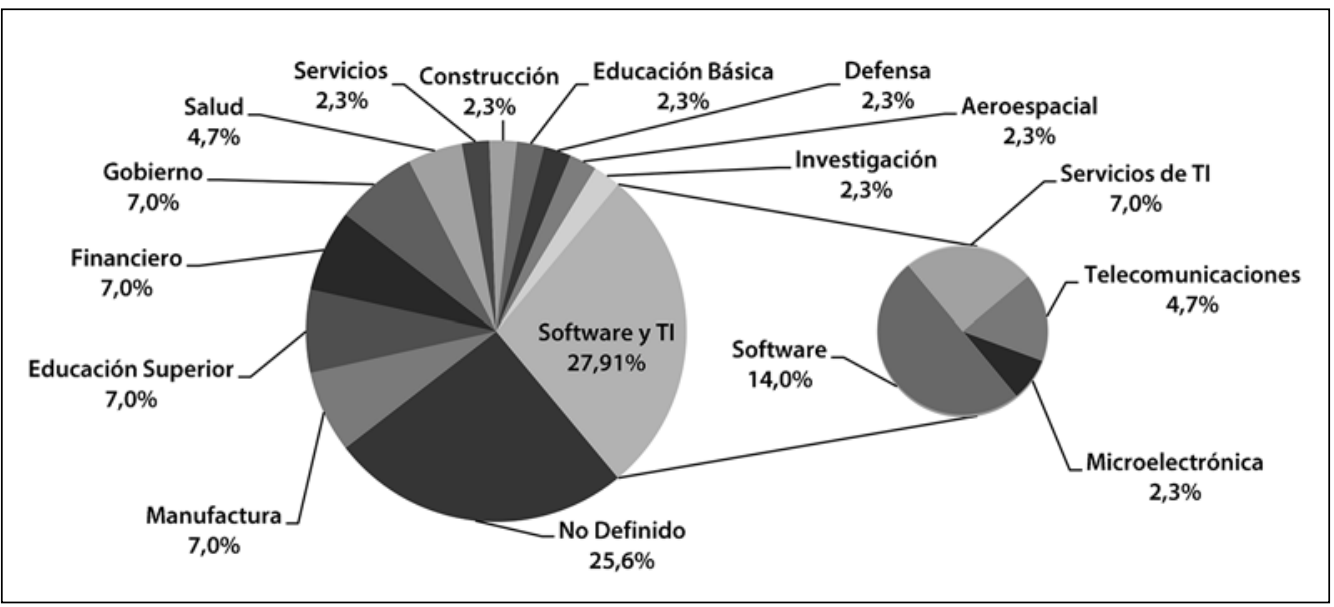

Fuente: Elaboración propia.

Otro de los aspectos tenidos en cuenta dentro del análisis de contenido fue el hecho de si los modelos de evaluación se centran en los procesos de GC en las organizaciones. Aquí se encontró que ocho modelos se centran en la evaluación de procesos de GC, y en algunos casos toman como referente el modelo de evaluación de procesos de software CMMI (Capability Maturity Model Integration - CMMI) propuesto por el Instituto de Ingeniería de Software de la Universidad Carnegie Mellon (CMMI Product Team, 2010). Como se ve en la Tabla 8, al tomar como referencia las escuelas de GC, se observa que hay una concentración en los aspectos relacionados con las escuelas de Sistemas, Ingeniería y Organizacional y, por el contrario, los postulados de las escuelas Cartográfica y Espacial no tienen lugar dentro de los modelos. Así mismo, respecto de las capacidades de GC incluidas, 
se ve que la capacidad de infraestructura de tecnología está incluida en los ocho modelos. Por último, se ve que las propuestas de Hung \& Shou (2005) y Khatibian et al. (2010), son las que incluyen aspectos de cuatro de las siete escuelas de GC y de seis de las siete capacidades de GC.

Por otra parte, se encontró que tres de los modelos fueron aplicados en organizaciones desarrolladoras de software, sin embargo, en el trabajo de Hsieh et al. (2009), no se brindan detalles de la aplicación pues simplemente se dice que en el grupo de 30 compañías en donde se utilizó el modelo, una era de la industria del software. Así mismo, en el trabajo de Khatibian et al. (2010), se describen la aplicación de un instrumento, pero no se dan detalles sobre las características de la organización objeto de estudio, ni se evidencian argumentos relativos a los enfoques metodológicos o a la cultura de la organización. Además, estos tres modelos no incluyeron características organizacionales determinantes del estilo de GC implementado, como el tamaño de la organización o el enfoque para el desarrollo de software (tradicional-ágil).

\section{Tabla 8}

\section{Caracterización de modelos de evaluación de procesos de GC}

\begin{tabular}{|c|c|c|c|c|c|c|c|c|c|c|c|c|c|c|c|c|}
\hline \multirow[b]{2}{*}{ Modelo } & \multirow[b]{2}{*}{ Año } & \multirow[b]{2}{*}{$\begin{array}{c}\text { Aplicado } \\
\text { en la } \\
\text { industria } \\
\text { del } \\
\text { software }\end{array}$} & \multicolumn{7}{|c|}{$\begin{array}{l}\text { Escuelas de GC } \\
(\text { Earl, 2001) }\end{array}$} & \multicolumn{7}{|c|}{$\begin{array}{l}\text { Capacidades de GC } \\
\text { (Gold et al., 2001) }\end{array}$} \\
\hline & & & 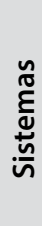 & 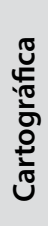 & 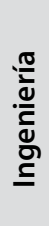 & 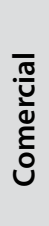 & 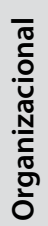 & $\begin{array}{l}\overline{.0} \\
\frac{\pi}{\mathscr{0}} \\
\frac{0}{4} \\
\text { ய }\end{array}$ & 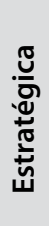 & 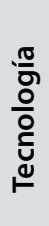 & $\frac{\frac{0}{3}}{\frac{2}{3}}$ & 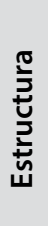 & 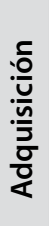 & 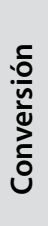 & 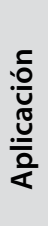 & 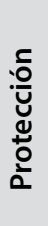 \\
\hline $\begin{array}{l}\text { (J.-H. Lee \& } \\
\text { Kim, 2001) }\end{array}$ & 2001 & - & $X$ & - & $X$ & - & $X$ & - & - & $X$ & - & - & $X$ & $x$ & $X$ & - \\
\hline $\begin{array}{l}\text { (Hung \& } \\
\text { Shou, 2005) }\end{array}$ & 2005 & - & $x$ & - & $X$ & $X$ & $X$ & - & - & $X$ & $X$ & $X$ & $x$ & $\mathrm{X}$ & $X$ & - \\
\hline $\begin{array}{l}\text { (Pee et al., } \\
\text { 2006) }\end{array}$ & 2006 & - & $x$ & - & $X$ & - & $X$ & - & - & $X$ & - & $X$ & - & $X$ & - & - \\
\hline (Li \& Li, 2008) & 2008 & & $X$ & - & $X$ & - & - & - & - & $X$ & - & - & - & $X$ & $X$ & - \\
\hline $\begin{array}{l}\text { (Hsieh et al., } \\
\text { 2009) }\end{array}$ & 2008 & $x$ & $x$ & - & $X$ & - & $X$ & - & - & $X$ & $X$ & - & $x$ & - & - & - \\
\hline $\begin{array}{l}\text { (Goldoni \& } \\
\text { Oliveira, 2010) }\end{array}$ & 2010 & $x$ & $\mathrm{X}$ & - & $X$ & $X$ & $X$ & - & - & $X$ & - & $X$ & $X$ & $X$ & $X$ & $x$ \\
\hline $\begin{array}{l}\text { (Khatibian et } \\
\text { al., 2010) }\end{array}$ & 2010 & $X$ & $x$ & - & $X$ & - & $X$ & - & $x$ & $X$ & $\mathrm{X}$ & $X$ & $X$ & - & $X$ & - \\
\hline (Tseng, 2010) & 2010 & - & - & - & - & $X$ & - & - & - & $X$ & - & - & - & - & $X$ & $X$ \\
\hline
\end{tabular}

Fuente: Elaboración propia. 


\section{Discusión}

Como se ve en los resultados, hay una gran variedad de propuestas para evaluar la GC en las organizaciones. Sin embargo, se encuentran varias brechas en el conocimiento en el área. En primer lugar, se encontró que el 69,8\% de los modelos centran la evaluación en los resultados y, solamente, el 18,6\% se enfoca en los procesos. Además, la mayoría de los modelos tiene una estructura sustentada en métodos de análisis cuantitativo y únicamente el 14\% propone una estructura orientada a la mejora y madurez de la GC en la organización. Esto indica que el interés principal de la evaluación es obtener calificaciones que permitan juzgar el estado de la GC en la organización, en lugar de asumir una función formativa. Por otra parte, la gran mayoría de los modelos fue construida a partir de una revisión de literatura y luego fueron puestos a prueba en organizaciones, y solamente 11 de los 43 modelos fueron construidos utilizando un método de intervención organizacional. Esto evidencia la tendencia hacia el enfoque positivista, en lugar del enfoque cualitativo, que, teniendo en cuenta la naturaleza del fenómeno en estudio, enriquecería más el contenido de los modelos (Bjørnson \& Dingsøyr, 2008; Checkland \& Holwell, 1998).

Sobre el contenido de la evaluación, se encontró que las escuelas Organizacional, Sistemas, e Ingeniería, aparecen en más del 70\% de los modelos. Además, se evidencia mayor interés en aspectos de la escuela Comercial, sin llegar a ser un consenso. Sin embargo, se identifican brechas pues no se incluyen aspectos de la escuela Espacial, y se abordan, muy someramente, los aspectos de las escuelas Cartográfica y Estratégica. Sin embargo, no se encontró un solo modelo que incluyera aspectos de todas las escuelas de GC. En este sentido, se encontró que el máximo número de escuelas incluidas en un modelo es cuatro, y los siete modelos que tienen ese contenido (Goldoni \& Oliveira, 2010; Hung \& Shou, 2005; Khatibian et al., 2010; Suo et al., 2008; Wen, 2009; Xia et al., 2010; Zaim et al., 2007), se centran en las escuelas Organizacional, Ingeniería, Sistemas y Comercial.

Así mismo, al tomar como referencia los planteamiento de las capacidades de GC (Gold et al., 2001), se encontró que la capacidad de infraestructura relacionada con la cultura organizacional de aprovechamiento del conocimiento es la menos abordada. Además, se evidencia que las capacidades de procesos de conversión y protección del conocimiento no son abordadas por la mayoría de modelos. Así mismo, se encuentran dos modelos (Fan et al., 2009; Zaim et al., 2007) que cubren las siete capacidades de GC, lo cual se explica porque tomaron como referencia el trabajo de Gold et al (Gold et al., 2001) para construir la estructura del modelo. Sin embargo, estos dos modelos no establecen con detalle los elementos constituyentes del modelo de evaluación, y están orientados exclusivamente a la evaluación cuantitativa.

Otra dimensión de la revisión mostró la diversidad de contextos de aplicación que tuvieron los modelos durante su construcción o su validación. Sin embargo, en el 25,6\% de los modelos no se hace distinción sobre el tipo de organización y el sector económico al que pertenece. Esto puede explicarse al revisar los métodos de investigación utilizados en la formulación de los modelos, pues al estar ubicados en el enfoque positivista, poseen la intención subyacente de demostrar que los 
resultados son repetibles en cualquier contexto de aplicación. No obstante, se ve que el sector de software y servicios de TI ha sido el contexto de aplicación más trabajado. Esto, sumado al hecho de que los modelos construidos y aplicados en la industria del software y servicios de TI han surgido durante los últimos tres años, muestra la importancia que tiene la temática de evaluación de GC para este sector de la economía.

Sin embargo, al analizar la estructura y contenido de los modelos utilizados en el sector de software y servicios de TI se encuentra que la aplicación en el contexto de una organización desarrolladora de software no incluyen características específicas de la organización, formadas a partir de la naturaleza y el enfoque asumido para realizar su actividad principal: desarrollar software. Esta distinción sobre el enfoque metodológico es un aspecto importante pues en la actualidad existen tendencias fuertes, generadas a partir del surgimiento y rápido posicionamiento de los métodos ágiles para el desarrollo de software, que han transformado la práctica cotidiana de las organizaciones desarrolladoras de software. Esto es importante como quiera que el origen de estos métodos, formulados como reacción a los métodos tradicionales, se sustenta en la idea construir software en un mundo impredecible centrándose en las personas y su creatividad en lugar de los procesos rígidamente definidos Dybå \& Dingsøyr (2008). En este sentido, la idea de Dingsøyr, Bjornson, \& Shull (2009), de que la forma en que se implementa la GC en una organización desarrolladora de software está determinada por el enfoque metodológico asumido, debe contemplarse como aspecto esencial de las propuestas que pretendan definir la forma en que se deben evaluar los procesos de GC en una organización desarrolladora de software, con el fin de lograr mayor efectividad en la aplicación de los modelos.

Ahora bien, al centrar el análisis en aquellos modelos centrados en la evaluación de procesos de GC, se encontraron varios aspectos susceptibles de mejora. Por ejemplo, no están incluidos aspectos de las escuelas Cartográfica, Comercial, Espacial y Estratégica. También, al revisar la ubicación del contenido evaluado con respecto a las capacidades de GC, se evidencia que, en conjunto, los modelos contemplan aspectos de todas las capacidades, con excepción de la capacidad relacionada con la protección del conocimiento organizacional. Esta brecha resulta, particularmente, problemática en el contexto de la industria del software toda vez que es una industria intensiva en conocimiento. Por lo tanto, si una organización desarrolladora de software que ha implementado estrategias ubicadas en las escuelas que no se incluyen en el modelo, desea utilizarlo como base para la determinación de las capacidades de procesos o la mejora de procesos, no va a obtener la caracterización completa de sus procesos.

En síntesis, se identifican tres aspectos percibidos como problemáticos. En primer lugar, los modelos de evaluación de la GC propuestos en la literatura académica sobre GC no integran los diferentes enfoques desde los que se implementa la GC en una organización. Como segundo punto, en los modelos de evaluación enfocados en procesos de GC se excluye de su contenido aspectos de varias escuelas de GC y capacidades organizacionales de GC. Y en tercer lugar, la formulación y aplicación de los modelos de evaluación de GC en organizaciones de la industria del software no toma en cuenta características como el tamaño y la tendencia del enfoque metodológico para el desarrollo de software. 


\section{Conclusiones}

Esta revisión sistemática de literatura es un aporte inicial a la búsqueda de consensos en el campo de la Gestión de Conocimiento. Particularmente, se identificaron modelos de evaluación de la GC enfocados a diferentes tipos y objetos de evaluación. Los modelos identificados fueron sometidos a los análisis necesarios para dar respuesta a las preguntas de interés que motivaron el desarrollo de este trabajo, evidenciando el logro del objetivo trazado para este estudio.

Sumado a esto, los argumentos presentados en la discusión permiten formular varios cuestionamientos que sirven como motivación para desarrollar un programa de investigación sobre evaluación de GC, y en particular sobre la evaluación de procesos de GC en organizaciones desarrolladoras de software de los contextos colombiano y latinoamericano. El primer cuestionamiento se centra en la identificación de los aspectos que se deben incluir en la evaluación de procesos de GC en una organización desarrolladora de software. Sumado a esto, se debe indagar sobre qué características particulares de las organizaciones de la industria del software en Colombia y Latinoamérica, se deben tener en cuenta al definir el contenido de la evaluación de procesos de GC. Ahora bien, en el plano operacional de la evaluación, se debe resolver el cuestionamiento sobre cómo se debe realizar la evaluación de procesos de GC en una organización que desea determinar la capacidad de sus procesos, u orientar y priorizar la mejora de procesos. Así mismo, se debe definir qué herramientas metodológicas y tecnológicas se deben utilizar para facilitar la evaluación.

Por otra parte, y teniendo en cuenta que la evaluación de procesos no es un tema nuevo para la industria del software, se debe responder cómo se puede articular la evaluación de GC con otras iniciativas de evaluación de procesos guiadas por modelos basados en la norma ISO/IEC 15504, como la Integración del Modelo de Madurez de Capacidad (Capability Maturity Model Integration - CMMI) propuesto por el Instituto de Ingeniería de Software de la Universidad Carnegie Mellon (Software Engineering Institute - SEI), el Modelo de Mejora de Proceso del Software Brasileño - MPS.BR propuesto por la Asociación para la Promoción y la Excelencia del Software Brasileño - Softex, el Modelo de Procesos para la Industria del Software - MoProSoft propuesto como la norma técnica NMX-059/01-NYCE-2005 de la república de México, y el Modelo para la Mejora de Procesos para Fomentar la Competitividad de la Pequeña y Mediana Industria del Software de Iberoamérica - Competisoft.

\section{Agradecimientos}

Los autores expresan su agradecimiento al Departamento Administrativo de Ciencia, Tecnología e Innovación Colciencias por el apoyo brindado a través del programa "Generación del Bicentenario" (Convocatoria 511 del 2010) y a la Universidad del Magdalena por el apoyo brindado a través del Programa de Formación Avanzada para la Docencia. Estos programas financian los estudios doctorales del primer autor. 


\section{Referencias Bibliográficas}

ANANTATMULA, V., \& SHIVRAJ, K. (2006). Structuring the underlying relations among the knowledge management outcomes. Journal of Knowledge Management, 10(4), 25-42. doi:10.1108/13673270610679345

BJØRNSON, F. O., \& DINGSØYR, T. (2008). Knowledge management in software engineering: A systematic review of studied concepts, findings and research methods used. Information and Software Technology, 50(11), 1055-1068. doi:10.1016/j.infsof.2008.03.006

CHECKLAND, P., \& HOLWELL, S. (1998). Action Research: Its Nature and Validity. Systemic Practice and Action Research, 11(1), 9-21. doi:10.1023/A:1022908820784

CHEN, M.-Y., HUANG, M.-J., \& CHENG, Y.-C. (2009). Measuring knowledge management performance using a competitive perspective: An empirical study. Expert Systems with Applications, 36(4), 8449-8459. doi:10.1016/j. eswa.2008.10.067.

CHIN, K.-S., LO, K.-C., \& LEUNG, J.P. F. (2010). Development of user-satisfaction-based knowledge management performance measurement system with evidential reasoning approach. Expert Systems with Applications, 37(1), 366-382. doi:10.1016/j.eswa.2009.05.051

CHUA, A. Y. K., \& GOH, D. H. (2007). Measuring knowledge management projects: Fitting the mosaic pieces together. In Proceedings of the 40th Annual Hawaii International Conference on System Sciences 2007 - HICSS 2007 (p. 192b - 192b). Hawaii, USA: IEEE. doi:10.1109/HICSS.2007.337

CHUA, A. Y. K., \& GOH, D. H. (2008). Untying the knot of knowledge management measurement: A study of six public service agencies in Singapore. Journal of Information Science, 34(3), 259-274. doi:10.1177/0165551507084139

CMMI Product Team. (2010). CMMI ${ }^{\circledR}$ for Development, Version 1.3 (CMU/SEI-2010th-TR-033 ed.). Pittsburgh, PA, USA: Carnegie Mellon University.

DINGSøYR, T., BJORNSON, F. O., \& SHULL, F. (2009). What Do We Know about Knowledge Management? Practical Implications for Software Engineering. Software, IEEE, 26(3), 100-103.

DWIVEDI, Y. K., VENKITACHALAM, K., SHARIF, A. M., AL-KARAGHOULI, W., \& WEERAKKODY, V. (2011). Research trends in knowledge management: Analyzing the past and predicting the future. Information Systems Management, 28(1), 43-56.

DYBÅ, T., \& DINGSØYR, T. (2008). Empirical studies of agile software development: A systematic review. Information and Software Technology, 50(9-10), 833-859. doi:10.1016/j.infsof.2008.01.006

Earl, M. (2001). Knowledge Management Strategies: Toward a Taxonomy. J. Manage. Inf. Syst., 18(1), 215-233.

FAN, Z.-P., FENG, B., SUN, Y.-H., \& OU, W. (2009). Evaluating knowledge management capability of organizations: a fuzzy linguistic method. Expert Systems with Applications, 36(2), 3346-3354. doi:10.1016/j. eswa.2008.01.052

FANG, J., PEIDE, L., \& XIN, Z. (2007). The evaluation study of knowledge management performance based on Grey-AHP method. In Proceedings of the Eighth ACIS International Conference on Software Engineering, Artificial Intelligence, Networking, and Parallel/Distributed Computing- SNPD 2007 (Vol. 3, pp. 444-449). Qingdao, China: IEEE. doi:10.1109/SNPD.2007.534 
FREEZE, R. D., \& KULKARNI, U. (2005). Knowledge Management Capability Assessment: Validating a knowledge assets measurement instrument. In Proceedings of the 38th Annual Hawaii International Conference on System Sciences 2005 - HICSS 2005 (p. 251). Hawaii, USA: IEEE. doi:10.1109/HICSS.2005.375

GOLD, A. H., MALHOTRA, A., \& SEGARS, A. H. (2001). Knowledge management: an organizational capabilities perspective. Journal of Management Information Systems, 18(1), 185-214.

GOLDONI, V., \& OLIVEIRA, M. (2010). Knowledge management metrics in software development companies in Brazil. Journal of Knowledge Management, 14(2), 301-313. doi:10.1108/13673271011032427

GOONESEKERA, T., \& ZYNGIER, S. (2011). Measuring knowledge management maturity levels in organisation's production area using fuzzy linguistic variables. In Proceedings of the 44th Annual Hawaii International Conference on System Sciences 2011 - HICSS 2011 (pp. 1-10). Hawaii, USA: IEEE. doi:10.1109/HICSS.2011.304

GOUROVA, E., ANTONOVA, A., \& TODOROVA, Y. (2009). Knowledge audit concepts, processes and practice. WSEAS Transactions on Business and Economics, 6(12), 605-619.

HOLT, D. T., BARTCZAK, S. E., CLARK, S. W., \& TRENT, M. R. (2007). The development of an instrument to measure readiness for knowledge management. Knowledge Management Research and Practice, 5(2), 75-92. doi:10.1057/palgrave.kmrp.8500132

HSIEH, P. J., LIN, B., \& LIN, C. (2009). The construction and application of knowledge navigator model $\left(K N M^{T M}\right)$ : An evaluation of knowledge management maturity. Expert Systems with Applications, 36(2 PART 2), 4087-4100. doi:10.1016/j.eswa.2008.03.005

HUANG, M.-J., CHEN, M.-Y., \&YIEH, K. (2007). Comparing with your main competitor: the single most important task of knowledge management performance measurement. Journal of Information Science, 33(4), 416-434. doi:10.1177/0165551506076217

HUNG, Y.-H., CHOU, S.-C. T., \& TZENG, G.-H. (2011). Knowledge management adoption and assessment for SMEs by a novel MCDM approach. Decision Support Systems, 51(2), 270-291. doi:10.1016/j.dss.2010.11.021

HUNG, Y.-H., \& SHOU, S.-C. T. (2005). On constructing a knowledge management pyramid model. In Proceedings of the 2005 IEEE International Conference on Information Reuse and Integration - IRI - 2005 (pp. 1-6). Las Vegas, USA: IEEE. doi:10.1109/IRI-05.2005.1506440

JAFARI, M., REZAEENOUR, J., AKHAVAN, P., \& FESHARAKI, M. N. (2010). Strategic knowledge management in aerospace industries: a case study. Aircraft Engineering and Aerospace Technology, 82(1), 60-74. doi:10.1108/00022661011028128

KHATIBIAN, N., GHOLOI POUR, T. H., \& ABEDI JAFARI, H. (2010). Measurement of knowledge management maturity level within organizations. Business Strategy Series, 11(1), 54-70. doi:DOI: 10.1108/17515631011013113

KITCHENHAM, B. A., DYBÅ, T., \& JØRGENSEN, M. (2004). Evidence-based software engineering. In Proceedings - International Conference on Software Engineering (Vol. 26, pp. 273-281). Retrieved from http://www. scopus.com/inward/record.url?eid=2-s2.0-4544225764\&partnerlD=40\&md5=e0d3d4da6b47ef5e3d9c3 $11 \mathrm{e} 31 \mathrm{ba} 5 \mathrm{be} 3$

KITCHENHAM, B., \& CHARTERS, S. (2007). Guidelines for performing Systematic Literature Reviews in Software Engineering (No. EBSE 2007-001). UK: Keele University and Durham University Joint Report. Retrieved from http://www.dur.ac.uk/ebse/resources/guidelines/Systematic-reviews-5-8.pdf

KITCHENHAM, B., PEARL BRERETON, O., BUDGEN, D., TURNER, M., BAILEY, J., \& LINKMAN, S. (2009). Systematic literature reviews in software engineering - A systematic literature review. Information and Software Technology, 51(1), 7-15. doi:10.1016/j.infsof.2008.09.009 
KITCHENHAM, B., PRETORIUS, R., BUDGEN, D., PEARL BRERETON, O., TURNER, M., NIAZI, M., \& LINKMAN, S. (2010). Systematic literature reviews in software engineering - A tertiary study. Information and Software Technology, 52(8), 792-805. doi:16/j.infsof.2010.03.006

KURNIAWATI, A., \& ANTHONY, L. (2010). A framework for measuring performance faculty knowledge management program. In Proceedings of the 2010 IEEE International Conference on Industrial Engineering and Engineering Management (IEEM) (pp. 1087-1091). Macao, China: IEEE. doi:10.1109/IEEM.2010.5674251

LAN, T., ZHONG, R., \& DAI, H. (2008). Performance evaluation of tacit knowledge management based on multiple indicator ideal interval methods. In Proceedings of the 2008 IEEE International Conference on Service Operations and Logistics, and Informatics, IEEE/SOLI 2008 (Vol. 1, pp. 858-864). Beijing, China: IEEE. doi:10.1109/SOLI.2008.4686519

LEE, J.-H., \& KIM, Y.-G. (2001). A stage model of organizational knowledge management: a latent content analysis. Expert Systems with Applications, 20(4), 299-311. doi:10.1016/S0957-4174(01)00015-X

LEE, K. C., LEE, S., \& KANG, I. W. (2005). KMPI: Measuring knowledge management performance. Information and Management, 42(3), 469-482. doi:10.1016/j.im.2004.02.003

LI, H.-B., \& LI, L. (2008). DEA-based project knowledge management performance evaluation. In Proceedings of the 2007 International Conference on Management Science and Engineering, ICMSE'07 (pp. 1561-1566). Harbin, China: IEEE. doi:10.1109/ICMSE.2007.4422065

MEHTA, N. (2008). Successful knowledge management implementation in global software companies. Journal of Knowledge Management, 12(2), 42-56. doi:10.1108/13673270810859505

PEE, L., KANKANHALLI, A., \&TEAH, H. (2006). Development and Application of a General Knowledge Management Maturity Model. In Proceedings of the 2006 Pacific Asian Conferencen on Information Systemas - PACIS 2006 (p. Paper 12). Kualalumpur, Malasya: AIS. Retrieved from http://aisel.aisnet.org/pacis2006/12

QI, R.-G., \& LIU, S.-J. (2010). Research on comprehensive evaluation of enterprises knowledge management capabilities. In Proceedings of the 2010 International Conference on Management Science and Engineering, ICMSE 2010 (pp. 1031-1036). Melbourne, Australia: IEEE. doi:10.1109/ICMSE.2010.5719925

RAZI, M. J. M., \& KARIM, N. S. A. (2010). Assessing knowledge management readiness in organizations. In Proceedings of the 2010 International Symposium on Information Technology-System Development and Application and Knowledge Society, ITSim'10 (Vol. 3, pp. 1543-1548). Kualalumpur, Malasya: IEEE. doi:10.1109/ ITSIM.2010.5561631

SERENKO, A., BONTIS, N., BOOKER, L., SADEDDIN, K., \& HARDIE, T. (2010). A scientometric analysis of knowledge management and intellectual capital academic literature 1994. Journal of Knowledge Management, 14(1), 3 - 23. doi:10.1108/13673271011015534

SMITS, M., \& DE MOOR, A. (2004). Measuring knowledge management effectiveness in communities of practice. In Proceedings of the 37th Annual Hawaii International Conference on System Sciences, 2004 (HICSS'04) (Vol. 37, pp. 3677-3685). Hawaii, USA: IEEE. doi:10.1109/HICSS.2004.1265570

SUO, B., WANG, J.-B., DONG, F., \& ZHAO, Z. (2008). The fuzzy evaluation on enterprise knowledge management capability based on knowledge audit. In Proceedings of the 2008 IEEE International Conference on Service Operations and Logistics, and Informatics, IEEE/SOLI 2008 (Vol. 1, pp. 792-796). Beijing, China: IEEE. doi:10.1109/SOLI.2008.4686506

TSENG, M.-L. (2010). Using linguistic preferences and grey relational analysis to evaluate the environmental knowledge management capacity. Expert Systems with Applications, 37(1), 70-81. doi:10.1016/j. eswa.2009.05.020 
TSENG, M.-L. (2011). Using a hybrid MCDM model to evaluate firm environmental knowledge management in uncertainty. Applied Soft Computing Journal, 11(1), 1340-1352. doi:10.1016/j.asoc.2010.04.006

WANG, J., FANG, N. G., \& WANG, X. (2009). The empirical study on performance evaluation of knowledge management based on information entropy and osculating value. In Proceedings of the 2009 Asia-Pacific Conference on Information Processing, APCIP 2009 (Vol. 1, pp. 157-160). Shenzhen, China: IEEE. doi:10.1109/ APCIP.2009.48

WANG, Y., \& ZHENG, J. (2010). Knowledge management performance evaluation based on triangular fuzzy number. In Procedia Engineering - Proceedings of the 2010 Symposium on Security Detection and Information Processing (Vol. 7, pp. 38-45). China: Elsevier. doi:10.1016/j.proeng.2010.11.006

WEI, J.-Y., \& BI, R. (2008). Knowledge management performance evaluation based on ANP. In Proceedings of the 7th International Conference on Machine Learning and Cybernetics, ICMLC (Vol. 1, pp. 257-261). Kunming, China: IEEE. doi:10.1109/ICMLC.2008.4620414

WEN, Y.-F. (2009). An effectiveness measurement model for knowledge management. Knowledge-Based Systems, 22(5), 363-367. doi:10.1016/j.knosys.2009.02.007

XIA, L., PAN, J., CHEN, S., \& ZHAO, Y. (2010). Research on government knowledge management capability based on fuzzy comprehensive evaluation. In Proceedings of the 2nd IEEE International Conference on Information Management and Engineering - ICIME 2010 (Vol. 2, pp. 534-538). Chengdu, China: IEEE. doi:10.1109/ ICIME.2010.5477676

YANG, N. (2010). Research on the evaluation of knowledge management performance of High-tech Enterprise. In Proceedings of the 2010 IEEE 2nd Symposium on Web Society, SWS 2010 (pp. 462-465). Beijing, China: IEEE. doi:10.1109/SWS.2010.5607405

ZAIM, H., TATOGLU, E., \& ZAIM, S. (2007). Performance of knowledge management practices: a causal analysis. Journal of Knowledge Management, 11(6), 54-67. doi:10.1108/13673270710832163

ZHANG, H., \& HE, Y. (2010). Manufacturing knowledge management performance evaluation based on grey fuzzy evaluation. In Proceedings of the 2010 International Conference on Environmental Science and Information Application Technology, ESIAT 2010 (Vol. 3, pp. 472-475). Wuhan, China: IEEE. doi:10.1109/ ESIAT.2010.5568310

ZHANG, Q.-P., \& SHAN, W. (2007). Research on enterprise tacit knowledge management performance appraisal based on artificial Neural Networks. In Proceedings of the 2006 International Conference on Management Science and Engineering, ICMSE'06 (13th) (pp. 1333-1337). Lille, France: IEEE. doi:10.1109/ICMSE.2006.314237

ZHENG, W., \& HU, Y. (2009). Grey evaluation method of knowledge management capability. In Proceedings of the 2009 2nd International Workshop on Knowledge Discovery and Data Mining, WKKD 2009 (pp. 256-260). Moscow, Russia: IEEE. doi:10.1109/WKDD.2009.205

ZHU, X.-Y., \& WU, Y.-L. (2010). Research on the evaluation method for enterprise knowledge management performance based on triangular fuzzy number. In Proceedings of the 2010 International Conference on EBusiness and E-Government, ICEE 2010 (pp. 1911-1914). Guangzhow, China: IEEE. doi:10.1109/ICEE.2010.483

ZHU, Z.-H., \& XUE, D., -W. (2009). Fuzzy evaluation model of accounting firm knowledge management performance. In Proceedings of the 2009 International Conference on Information Management, Innovation Management and Industrial Engineering, ICIII 2009 (Vol. 2, pp. 75-78). Xi'an, China: IEEE. doi:10.1109/ICIII.2009.176 